\title{
Effect of triaxial stress distribution upon roughness of brittle fracture surface
}

\author{
Noritaka Nakamura ${ }^{1}$, Tomoya Kawabata $^{1}$, Yasuhito Takashima ${ }^{2}$, Yuki Nishizono ${ }^{1}$ and \\ Fuminori Yanagimoto ${ }^{1}$
}

${ }^{1}$ The University of Tokyo, Japan

${ }^{2}$ Osaka University, Japan

\begin{abstract}
To observe the effect of stress triaxiality upon brittle fracture surface, we performed two types of experiments which differ in stress triaxiality. As a result, crack branch starting speed changes in two specimens and the speed was affected by stress triaxiality. In bending condition, branch starting speed is around $0.86 c_{\mathrm{r}}\left(c_{\mathrm{r}}\right.$ : Rayleigh wave speed), which is higher than that in tensile condition, $0.59 c_{\mathrm{r}}$. It was realized that in higher stress triaxiality, branching is easy to occur because in bending condition stress triaxiality is said to be lower. On the other hand, mirror-mist transition speed is not affected by stress triaxiality. By fracture surface observation, we proposed that branch occurs when microbranch grew. This proposition was supported by FEM calculation with microbranch model, it was proved that in bending condition microbranch is difficult to grow. Additionally, we proposed a qualitative explanation that microbranch is easy to grow when stress triaxiality is higher because growth of microbranch is affected by T-stress. It is since the phenomena is not on the main crack propagating plane.
\end{abstract}

\section{Introduction}

\subsection{Background}

As the result of the growth of demands for international transportation and energy, recent ships are in the higher risk of brittle fracture of their structural steel. Brittle fracture threats shipping since brittle cracks propagate rapidly and they lead ships into fatal collapse in a moment. When ships become larger so as to accomplish the larger capacity for transportation, the structural steel become thicker and it makes brittle cracks propagate easily. Additionally, when ships are used in environment with lower temperature, like Arctic Ocean, steel loses brittle toughness and it makes brittle cracks unlike to be arrested. Steel makers have tried hard to develop technologies give steel improved brittle toughness enough to stop propagating brittle cracks. For example, TMCP method, it is a technology of make steel stronger and tougher by controlling cooling rate in the stage of steel extension. However, this method has a limit of maximum thickness around $100 \mathrm{~mm}$, and some parts of structure in enormous container ships have already reached $100 \mathrm{~mm}$. Other example of 
technologies is to blend rare metals such as nickel into steel. Although this method can improve brittle toughness without technical difficulties, price of structural steel gets higher and unstable. Hence it can be said that there is scarce stretch of improvement of brittle toughness with existing technologies. In order to develop shipping further, demands for completely new concept of technology to improve brittle toughness is in urgent demand.

Now we propose that fracture roughness may be used to improve brittle toughness. It is thought by an energy balance in crack propagation that rougher fracture surface needs more energy dissipated to form and it results in decrease of force which propagates brittle crack. Thus rougher surface makes the crack difficult to grow. If we could make the fracture surface rougher intendedly, we also could make brittle crack controlled and prevent structures from fatal collapse.

\subsection{Related research}

In the past, many researches have performed about fracture surface roughness. Commonly, fracture surface becomes rougher when crack speed is higher. In relatively low crack speed, smooth surface generally called "mirror" can be seen. When crack speed become higher, rough surface called "mist" appears. In many materials transition from mirror to mist appear clearly and fracture surface roughness changes drastically. In even higher crack speed, sometimes crack would branch or extremely rough surface called "hackle" would appear. It is generally thought that fracture surface roughness is only affected by crack speed. It means that crack surface roughness is completely determined by crack speed. Crack model proposed by Freund[1] is one of the famous theories to explain fracture surface roughness. It is proposed in linear-elastic dynamic fracture mechanics that when in high crack speed over about 0.6 times of Rayleigh wave speed, the angle showing maximum of hoop stress around the running crack tip changes from zero degree to a finite angle. But existing theories cannot explain the actual phenomena. Kobayashi[2] summarized crack branching speed from past experiments. They are mainly less than the speed of Freund[1]'s theoretical boundary speed. Furthermore, the crack branching speeds differs in different materials.

Some scientists insist that this contradiction between theories and experiments comes from that in actual crack propagation fracture surface roughness is not determined only by crack speed and there might be other factors affect it, in contrast of the general opinion that crack surface roughness is completely determined by crack speed. One of the proposed factors is stress triaxiality around the crack tip. Stress triaxiality is defined by the following equation and it is a parameter which is said to affect the initiation of brittle crack. Tonsho[3] insisted that it also affect brittle crack branching.

$$
\begin{aligned}
& \boldsymbol{\eta}=\boldsymbol{\sigma}_{\mathrm{m}} / \boldsymbol{\sigma}_{0} \\
& \boldsymbol{\sigma}_{\mathrm{m}}=\left(\boldsymbol{\sigma}_{1}+\boldsymbol{\sigma}_{2}+\boldsymbol{\sigma}_{3}\right) / 3 \\
& \boldsymbol{\sigma}_{0}=\sqrt{ }\left[\left(\boldsymbol{\sigma}_{1}-\boldsymbol{\sigma}_{2}\right)^{2}+\left(\boldsymbol{\sigma}_{1}-\boldsymbol{\sigma}_{2}\right)^{2}+\left(\boldsymbol{\sigma}_{1}-\boldsymbol{\sigma}_{2}\right)^{2}\right] / \sqrt{2}
\end{aligned}
$$

\subsection{Objective}

The objective of this research is to confirm the effect of stress triaxiality upon fracture surface roughness, which is an extensive hypothesis of that of proposed by Tonsho[3]. It consequences into explication of the mechanism of fracture roughness growth and moreover intensive utilization of rougher fracture to improve brittle toughness in the practical structures. Fig. 1 is an example of intensive utilization of crack branching. By 
positioning an element which promote brittle crack branching, crack branches on the aimed place and the crack would be robbed its energy to propagate and it would be led into deceleration and arrest.

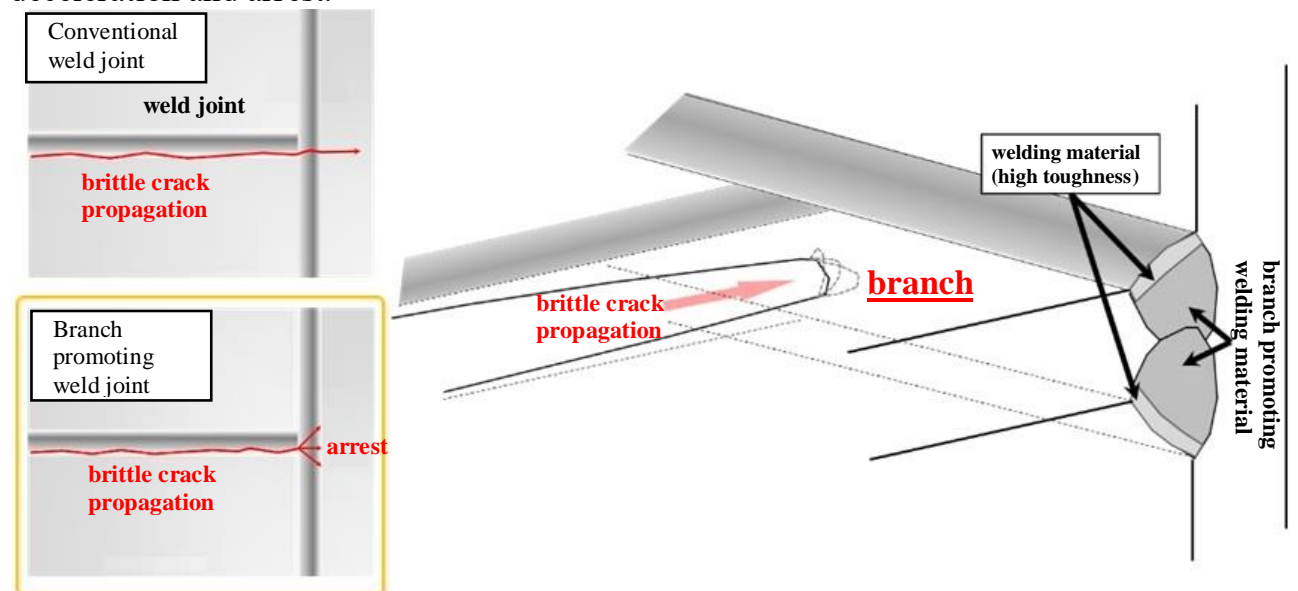

Fig. 1. One example of arresting promotion using branching

\section{Experiments}

\subsection{Overview}

We operated brittle fracture experiments with two types of specimens, tensile and bending specimens. Then, we compared the relation between fracture roughness and crack speed in two types of specimens. Since it had been proved that stress triaxiality differs in two types of specimens[4], effect of stress triaxiality upon fracture surface roughness can be observed by comparing results from these experiments. Fig.2 shows the forms of two types of specimens. Although as shown in introduction, this research finally treat with steel material, in these experiments we used resin materials. Using resin materials benefits in some points. They are amorphous and do not have crystal microstructures and suitable to observe principal mechanisms about forming fracture surface roughness. They are clear, so that it is easy to observe microcracks and branches formed internal of specimens. Additionally, in resin materials brittle fracture easily initiates even without cooling and suitable for a series of systematic experiments involving comparing. In these experiments, we used three species of resin materials from two categories as followings, PCET1600(Poly-carbonate), Comoglas(PMMA), Delaglas(PMMA). Crack speed was measured by recording the time that singular stress field near crack tip passes with sticked strain gauges on the surface of the specimens. Then, correspondence between crack speed and fracture surface is checked. We checked two boundary crack speeds which clearly changing fracture surface roughness, mirror-mist transition speed and branch starting speed. If these boundary crack speed differs between two types of specimens in the same material, it can be concluded that stress triaxiality affects fracture surface roughness. 


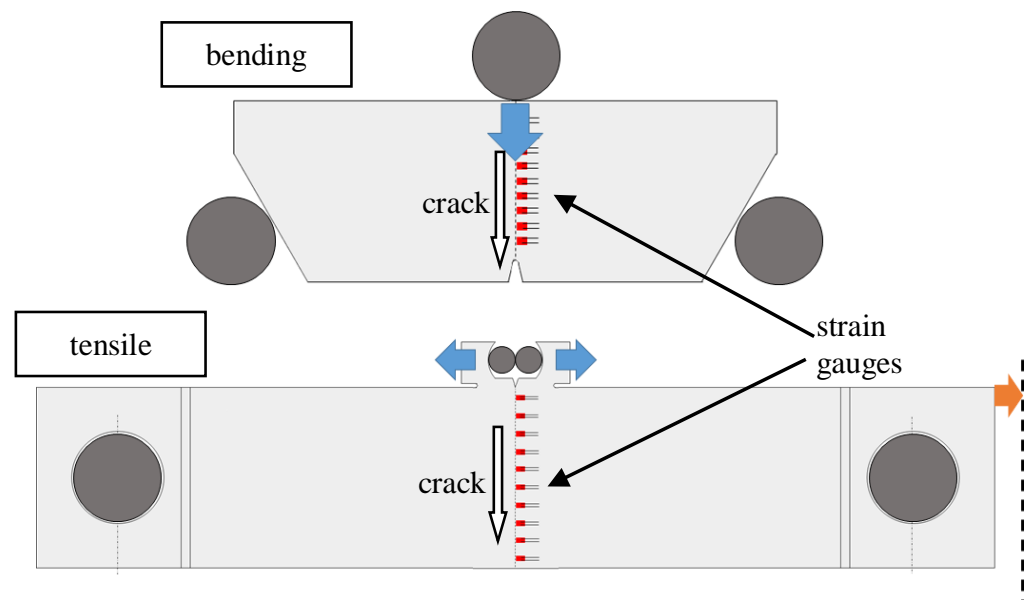

Fig. 2. Two types of specimens

\subsection{Result}

\subsubsection{Mirror-mist transition speed}

Fig. 3 is bending and tensile crack speed history in PCET1600 specimens. The position that mirror-mist transition occurred is shown in the figure. In bending specimens the transition occurred around $280 \mathrm{~m} / \mathrm{s}\left(0.35 c_{\mathrm{r}}\right)$ of crack speed. In tensile specimens though there were not observed the transition on the specimen, when crack speed was $300 \mathrm{~m} / \mathrm{s}\left(0.37 c_{\mathrm{r}}\right)$ the surface was mist and when $240 \mathrm{~m} / \mathrm{s}\left(0.30 c_{\mathrm{r}}\right)$ was mirror. Thus it can be said there was no clear effect of stress triaxiality upon mirror-mist transition speed.
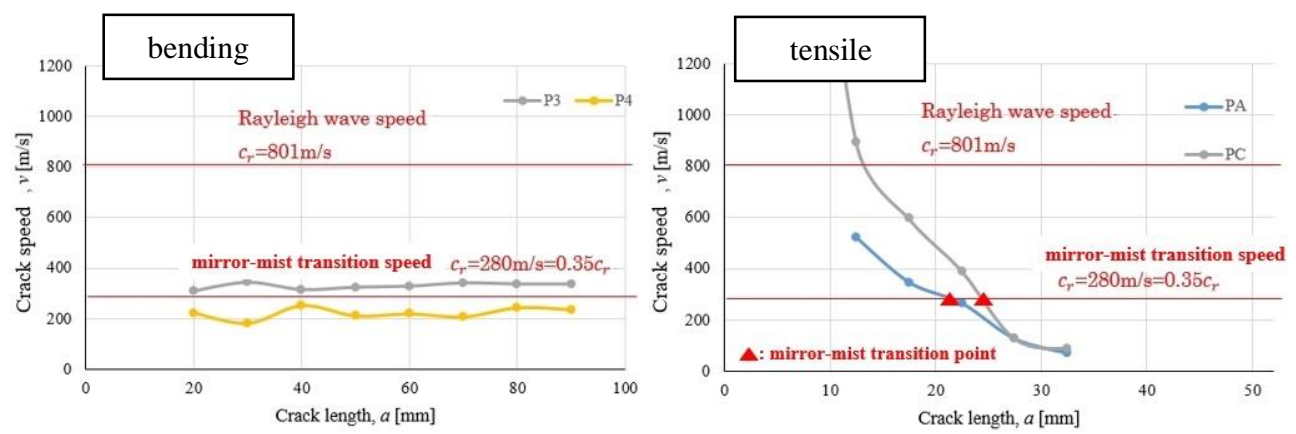

Fig. 3. Comparison of mirror-mist transition speed between two types of specimens (PCET1600)

\subsubsection{Branch starting speed}

Fig. 4 is bending and tensile crack speed history in PCET1600 specimens. The position that branch occurred is shown in the figure. In tensile specimen branching could be seen when crack speed was larger than $650 \mathrm{~m} / \mathrm{s}\left(0.59 c_{\mathrm{r}}\right)$. However, in bending specimens branching could only be seen when crack speed was larger than $950 \mathrm{~m} / \mathrm{s}\left(0.86 c_{\mathrm{r}}\right)$. Consequently, in bending specimen it was more difficult for brittle crack to branch than in tensile specimen. Here, it is proved that around notch of bending specimen, where branching occurred only in high crack speed, stress triaxiality is considerably small[4]. As a result, it can be concluded that high stress triaxiality promotes branching. 

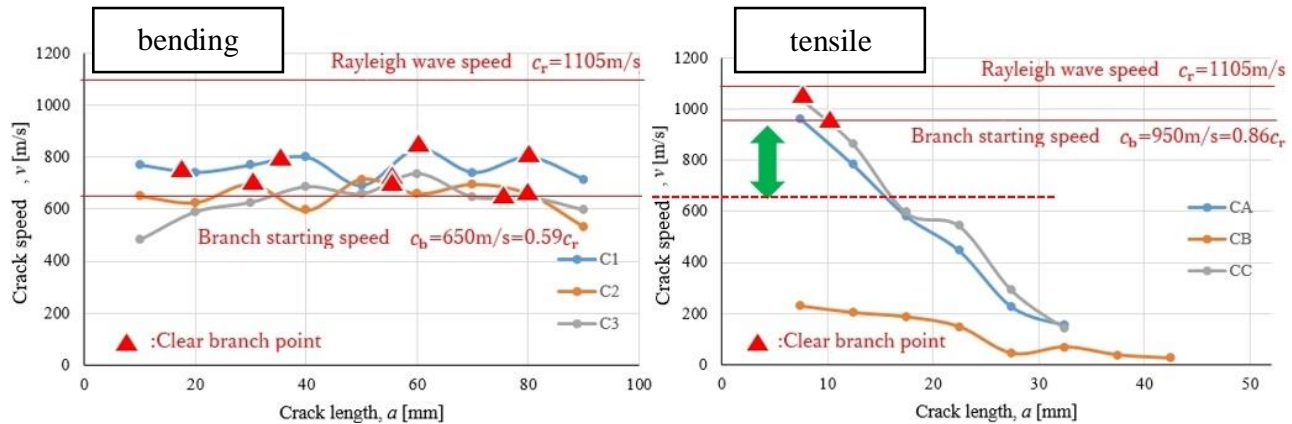

Fig. 4. Comparison of branch starting speed between two types of specimens (Comoglas)

\section{Fracture surface observation}

By making fracture surface from these experiments observed carefully by a laser microscope, we tried to elucidate micromechanisims of forming of fracture surface.

\subsection{Mirror region}

By observing mirror surface, numerous parabolic patterns could be seen. These patterns are caused when microcrack generated ahead of main crack tip grew and coalesced with main crack. Microcrack can be thought to be generated when crack speed is small, even in $200 \mathrm{~m} / \mathrm{s}$. The size of parabola pattern is affected by crack speed, and not affected by stress triaxiality. When in larger crack speed the size of parabola pattern is larger. The size of parabola patterns is greatly affected by material. In PCET1600 and Comoglas, it was at most $5 \mu \mathrm{m}$, In Delaglas it could reach up to $150 \mu \mathrm{m}$.

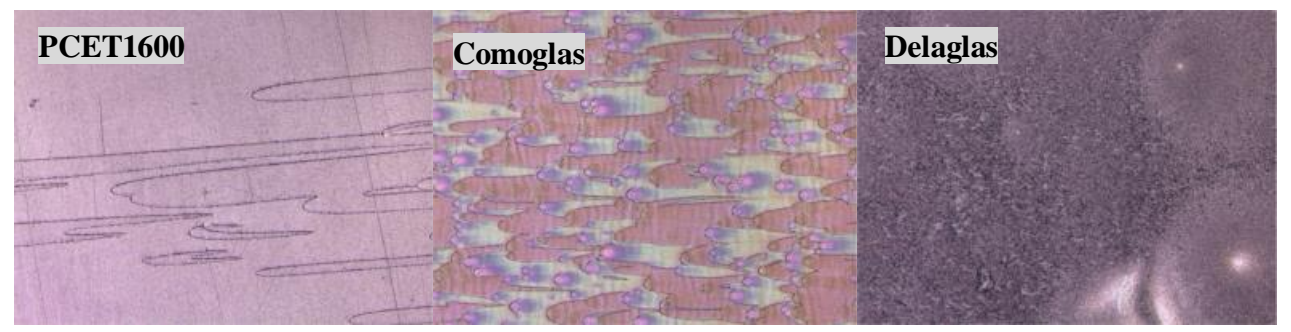

Fig. 5. Parabola patterns in each materials

\subsection{Mist region}

By observing mist surface, periodic patterns of rough and smooth surfaces could be seen in relatively low crack speed in mist region. When crack speed get larger, the proportion that rough surface occupies get larger too, and finally whole surface would be rough surface. The interval of periodic pattern is greatly affected by material. In PCET1600 it was around $16 \mu \mathrm{m}$, however in Comoglas it was around $200 \mu \mathrm{m}$. The interval was not affected by stress triaxiality. In contrast to the size of parabola pattern, the interval of periodic pattern was not affected by crack speed.

By observing mist surface, many microbranchings could be seen when crack speed is much smaller than crack branching speed. Thereby it was thought that branch occurs when microbranching grew. 


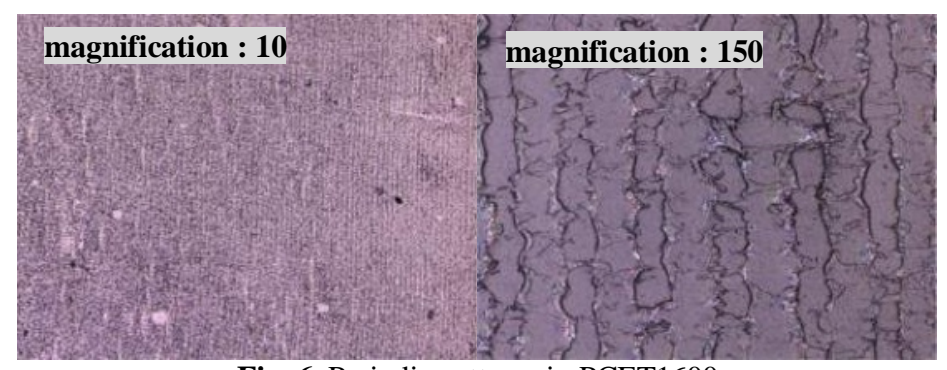

Fig. 6. Periodic patterns in PCET1600

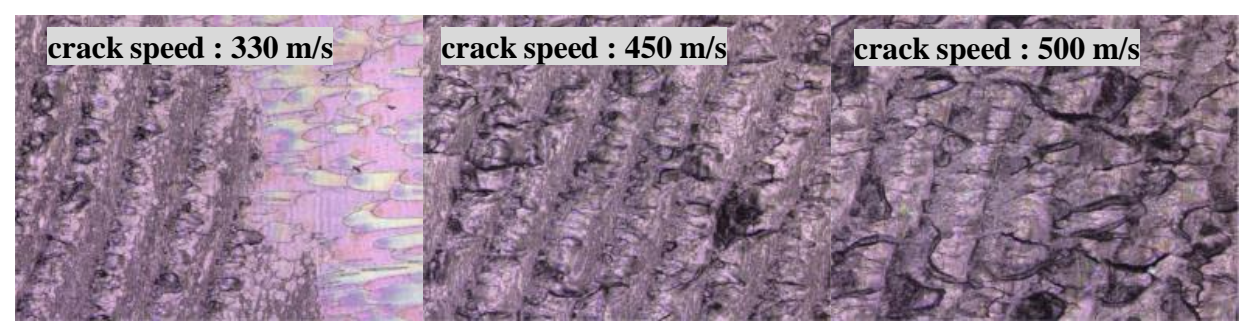

Fig. 7. Periodic patterns in different crack speeds (Comoglas)

\subsection{Fracture surface and stress triaxiality}

In Fig. 8 the correspondence between fracture surface and crack speed is summarized in each material and type of specimen. Briefly, only branch starting speed was affected by tress triaxiality. Generation of microcrack and microbranch, also growth of microcrack are not affected by stress triaxiality. Just when discussing about these phenomena, it would be true that fracture surface roughness is controlled only by crack speed. On the other hand, branching was affected by stress triaxiality and it could be said that in higher stress triaxiality condition, microbranch is easy to grow up.

As one qualitative explanation for result from the experiment that in higher stress triaxiality branch is easy to occur, and our proposition that branch occurs when microcrack grew, we propose an effect of T-stress. Now, T-stress is a stress tensor component on the direction of main crack propagation, and generally stress triaxiality get larger when T-stress is larger. All of the phenomena that were not affected by stress triaxiality, generation of microcrack and microbranch, and growth of microcrack, were phenomena only on the plane which main crack propagates. Thus these phenomena were not affected by T-stress. By contrast, existing microbranch is not on the plane which main crack propagates. It could be thought that these branched crack grew easily when T-stress is larger.

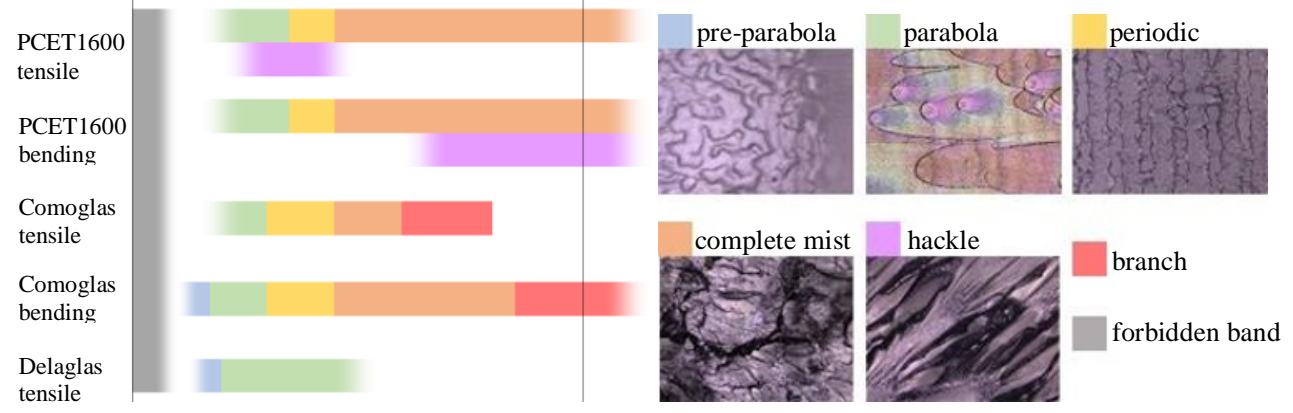

Fig. 8. Relation between crack speed and fracture surface patterns in each type of specimens 


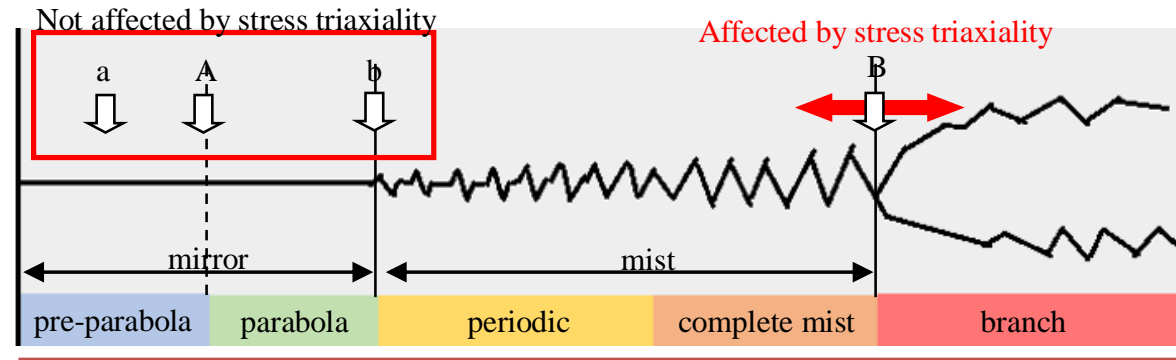

higher crack speed

Fig. 9. Relation between crack speed and fracture surface patterns in each type of specimens a: generation of microcrack A: growth of microcrack

b: generation of microbranch B: growth of microbranch

\section{FEM simulation}

\subsection{Overview}

By observing mist region, it was supposed that growth of microbranch causes branch. To confirm this concept, we performed an FEM calculation with branched crack. We loaded this model by bending and tensile condition and evaluated stress intensity factor (SIF) on the branched crack tip. SIF from this model was normalized by the SIF of crack with same length and without branching. Finally we compared the normalized SIF from bending and tensile conditions and demonstrate if in bending condition branched crack is really difficult to grow.

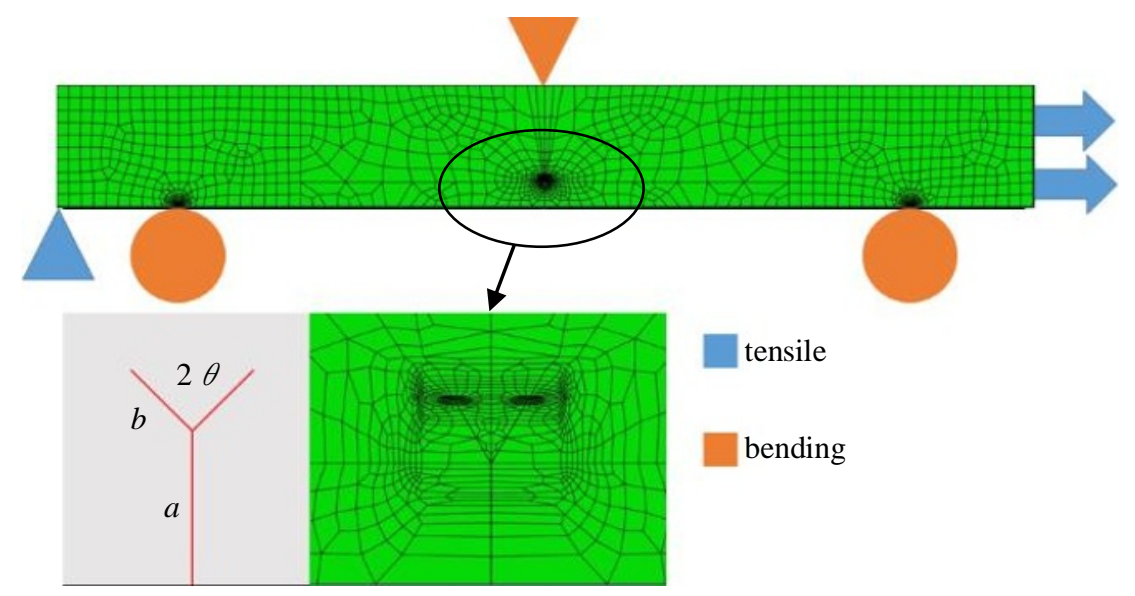

Fig. 10. Schematic image of FEM calculation model

\subsection{Result}

Result from various crack branching angle and branched crack length were plotted in Fig.11. Now, $F$ is rate of SIF in bending and tensile condition. When $F$ is smaller than 1, 
SIF is smaller in bending condition. It shows that when crack branching angle was around 60 deg., in bending condition normalized SIF is smaller than tensile condition. Here, it had been proved in linear-elastic dynamic fracture mechanics that crack branching angle would be around 60 deg., when crack speed is around $0.75 c_{\mathrm{r}}$, which microbranch was observed in. It can be said that this result from FEM calculation supported that in bending specimen branch could be seen only in high crack speed because microbranch was difficult to grow up.

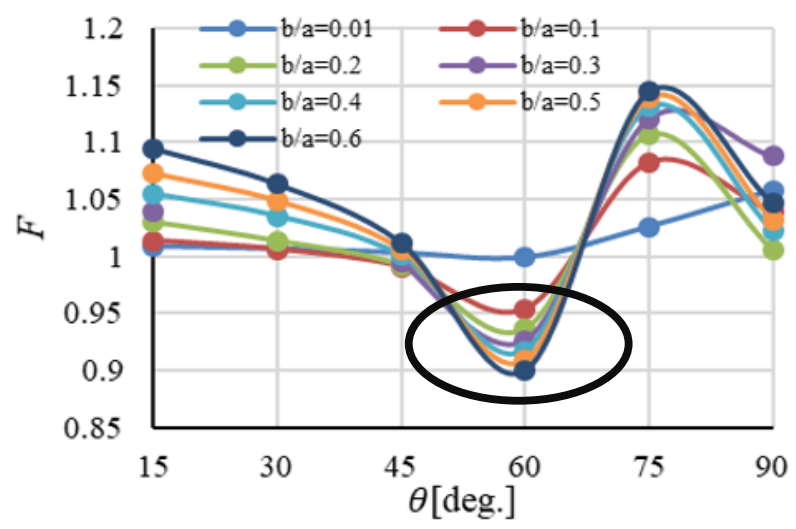

Fig. 11. Change of the rate of normalized stress intensity factors of branched crack between tensile and bending conditions, $F$ by $\theta$

\section{Conclusion}

In this research, it is concluded as following. It was experimentally indicated that the boundary condition of mirror-mist transition was not affected by stress triaxiality, but the condition of branching was affected by stress triaxiality and when stress triaxiality is larger branching is easy to occur. By observing fracture surface, we proposed that branching is caused by growth of microbranching, and only branching was affected by stress triaxiality, or T-stress, because these phenomena do not occur just on the plane which main crack propagates. FEM calculation supported these proposition of ours in that microbranch is difficult to grow up in the model with bending condition. Consequently, it can be said that we approached to achieve our objective, explication of the mechanism of fracture roughness growth and intensive utilization of rougher fracture to improve brittle toughness in the practical structures.

\section{References}

1. L.B. Freund, R.J. Clifton, On the uniqueness of elastodynamic solutions for running cracks, Journal of Elasticity 4, pp.293-299 (1974)

2. A. Kobayashi, M. Ramulu, A Dynamic Fracture Analysis of Crack Curving and Branching, International Journal of Fracture 27, Issue 3-4, pp.187-201 (1985)

3. F. Tonsho, Study on Crack Branching Criteria and Increase of Dissipation Energy for Brittle Crack Propagation in Steels (2017)

4. M. Toyoda, F. Minami, C. Ruggieri et al., Fracture Toughness Measurement for Fracture Performance Evaluation of Weldments, quarterly journal of the Japan Welding Society 11-3, pp.454-460 (1993) 